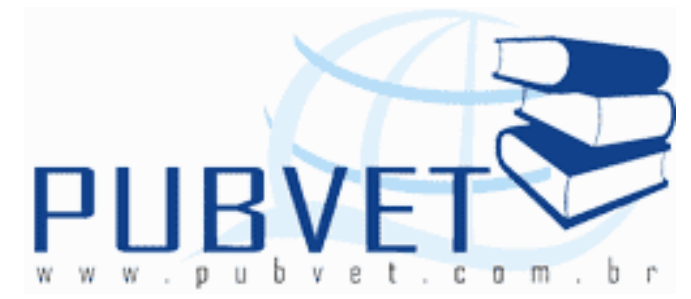

PUBVET, Publicações em Medicina Veterinária e Zootecnia.

\title{
Incidência de Leishmaniose Tegumentar Americana no Município de Timon/MA
}

\author{
Cielly Cristine Veras Alves ${ }^{1}$, Nilton Andrade Magalhães ${ }^{2}$, Gioto Ghiarone \\ Terto e Sousa ${ }^{3}$
}

${ }^{1}$ Zootecnista, Instituto de Ensino Superior Múltiplo, IESM, Timon/MA, Brasil. ${ }^{2}$ Docente, Instituto de Ensino Superior Múltiplo, IESM, Timon/MA, Brasil. ${ }^{3}$ Pós-Graduando em Ciência Animal, Universidade Federal do Piauí, UFPI, Teresina/PI, Brasil.

\section{Resumo}

Leishmaniose Tegumentar Americana (LTA) infecção zoonótica não contagiosa causada por protozoários do gênero Leishmania, que acomete pele e mucosas, podendo ser antropozoonose com a maioria dos infectados permanecendo assintomáticos. Estima-se que 350 milhões de pessoas estejam expostas ao risco com registro aproximado de dois milhões de novos casos. A pesquisa é um estudo retrospectivo, através do levantamento de casos registrados de LTA do município de Timon-MA, junto a Secretaria Municipal de Saúde e do Centro de Controle de Zoonoses no período de 2007 a 2011. O objetivo deste trabalho foi realizar um levantamento do número de casos de LTA e incidência flebotomínea no município, observando a relação dos espécimes capturados como potenciais vetores na participante do ciclo da doença. No período foram registrados 60 casos. $O$ ano de 2011 apresentou maior incidência com 22 
ALVES, C.C.V., MAGALHÃES, N.A. e SOUSA, G.G.T. Incidência de Leishmaniose Tegumentar Americana no Município de Timon/MA. PUBVET, Londrina, V. 6, N. 30, Ed. 217, Art. 1449, 2012.

casos registrados até o mês de setembro representando $36,67 \%$ de todas as ocorrências do município. O período que apresentou menor incidência foi 2008 e 2009 com seis casos (10\%) em cada ano. Dos notificados, 66,7\% eram do sexo masculino. No município os casos ocorreram mais em indivíduos com idade de 50 a 64 anos com 15 casos representando $25 \%$ da população em estudo. A captura de flebotomíneos refere-se ao período de agosto de 2006 a setembro de 2008, realizados nos bairros Loteamento Boa Vista, Parque Piauí, Centro Operário e Parque Alvorada, onde foram identificadas as espécies: Lutzomyia longipalpis, L. lenti, L. evandroi, L. termitophila, L. goiana e L. migonei.

Palavras-chave: Epidemiologia, leishmaniose humana, leishmaniose tegumentar.

\title{
Incidence of Tegumentary Leishmaniasis Americana in the municipality of Timon/MA
}

\begin{abstract}
Tegumentary leishmaniasis Americana (LTA) zoonotic infection not contagious disease caused by protozoa of the genus Leishmania, which affects the skin and mucous membranes, and may be antropozoonose with the most of those infected remained asymptomatic. It is estimated that 350 million people are at risk, with registration of approximately two million new cases. The search is a retrospective study, through the survey of registered cases of LTA Timon-MA, next to the Municipal Health and zoonosis control center in the period 2007 to 2011. The aim of this study was to conduct a survey of the number of cases of LTA and incidence flebotomínea in the municipality, observing the relationship of captured specimens as potential vectors in the end of the cycle of the disease. In the period have been recorded 60 cases. The year 2011 has a higher incidence with 22 cases entered until the month of September representing $36.67 \%$ of all occurrences of the municipality. The period that showed lower incidence was 2008 and 2009 with six cases (10\%) in each year.
\end{abstract}


ALVES, C.C.V., MAGALHÃES, N.A. e SOUSA, G.G.T. Incidência de Leishmaniose Tegumentar Americana no Município de Timon/MA. PUBVET, Londrina, V. 6, N. 30, Ed. 217, Art. 1449, 2012.

Notified of $66.7 \%$ were male. In the most cases occurred in individuals aged 50 to 64 years with 15 cases representing $25 \%$ of the population under study. The capture of phlebotomine sandflies refers to the period from August 2006 to September 2008, performed at the Boa Vista Subdivision neighborhoods, Piauí, workers ' Centre, where Park and Alvorada species have been identified: Lutzomyia longipalpis, I. lenti, I. I. evandroi termitophila, I. goiana and I. migonei.

Keywords: Epidemiology, Human leishmaniasis, tegumentary leishmaniasis.

\section{Introdução}

A Leishmaniose Tegumentar Americana (LTA) é uma doença infecciosa e não contagiosa causada por protozoários do gênero Leishmania, que acomete pele e mucosas. É uma infecção zoonótica, acometendo animais silvestres, podendo o homem ser envolvido secundariamente (BRASIL, 2010).

No Brasil, Moreira em 1895 identificou pela primeira vez a existência do botão endêmico dos países quentes, chamando "Botão da Bahia" ou "Botão de Biskra" (VALE et al., 2005). A confirmação de formas de Leishmanias em úlceras cutâneas e nasobucofaringeas ocorreram em 1909, quando Lindenberg encontrou o parasito em indivíduos que trabalhavam em áreas de desmatamentos na construção de rodovias no interior de São Paulo.

As leishmanioses são antropozoonoses consideradas de risco à saúde pública, representando um complexo de doenças com importante caráter clínico e diversidade epidemiológica. De acordo com os dados anuais da Organização Mundial da Saúde (OMS) estima-se que 350 milhões de pessoas estejam expostas ao risco com registro aproximado de dois milhões de novos casos. A LTA tem ampla distribuição mundial e no Continente Americano há registro de casos desde o extremo sul dos Estados Unidos até o norte da Argentina, com exceção do Chile e Uruguai (BRASIL, 2007).

Alguns animais domésticos, criados no domicílio ou peridomicílio (cães, equídeos, galinhas) e silvestres (roedores, gambás e outro marsupiais), são 
ALVES, C.C.V., MAGALHÃES, N.A. e SOUSA, G.G.T. Incidência de Leishmaniose Tegumentar Americana no Município de Timon/MA. PUBVET, Londrina, V. 6, N. 30, Ed. 217, Art. 1449, 2012.

incriminados como reservatório do agente causador da LTA que associado ao crescimento desordenado deve está contribuindo para sua expansão.

O município de Timon-MA apresenta características que propiciam o desenvolvimento e manutenção do ciclo da doença como áreas de invasão, acúmulo de matéria orgânica em áreas periurbanas, presença de animais que estão envolvidos na transmissão da doença como equídeos e cães entre outros.

O objetivo da pesquisa foi realizar levantamento do número de casos de LTA e a incidência de flebotomíneos no município de Timon-MA, avaliando os espécimes capturados como potenciais vetores participantes do ciclo da doença.

\section{Material e métodos}

A pesquisa é um estudo retrospectivo, quantitativo transversal. Realizado através do levantamento de dados de casos registrados de LTA do município de Timon-MA, junto a Secretaria Municipal de Saúde e do Centro de Controle de Zoonoses referente ao período de 2007 a 2011. Foi realizado também levantamento de espécies de flebotomíneos capturados no período de setembro de 2006 a agosto de 2007.

O município de Timon-MA está situado a $5^{\circ} 5^{\prime} 38^{\prime \prime}$ de latitude sul e 42050'13" de longitude oeste, mesorregião leste maranhense, clima equatorial, com temperaturas variando de $20^{\circ} \mathrm{C}$ a $40^{\circ} \mathrm{C}$. Estando $69 \mathrm{~km}$ acima do nível do mar, distante $426 \mathrm{~km}$ da capital São Luís por rodovia e, em linha reta, apenas $322 \mathrm{~km}$. Com área da unidade territorial $1743,228 \mathrm{~km}^{2}$. A população é de 155.460 habitantes e densidade de 89,18 hab./km² (IBGE, 2010).

Timon possui um clima quente $\mathrm{o}$ ano inteiro, com a temperatura média variando pouco no ano inteiro, onde podem às vezes superar os $40^{\circ} \mathrm{C}$, e a mínima raramente é inferior a $20^{\circ} \mathrm{C}$. Até mesmo o mês mais frio da cidade 
ALVES, C.C.V., MAGALHÃES, N.A. e SOUSA, G.G.T. Incidência de Leishmaniose Tegumentar Americana no Município de Timon/MA. PUBVET, Londrina, V. 6, N. 30, Ed. 217, Art. 1449, 2012.

(fevereiro), tem uma temperatura anual alta: máxima de $32^{\circ} \mathrm{C}$ e mínima de $22^{\circ} \mathrm{C}$. O mês mais quente é outubro e a temperatura média é de $37^{\circ} \mathrm{C}$ ao dia e $23^{\circ} \mathrm{C}$ à noite "às vezes de manhã" (WIKIPÉDIA, 2011).

A chuva influencia muito na temperatura da cidade, os meses mais frios (dezembro-abril) são os mais chuvosos, assim, a umidade na cidade e a água fria diminuem a temperatura, então nesses meses a temperatura vai ser mais baixa. Dentre esses meses, o mais chuvoso é abril, chove cerca de inacreditáveis $287 \mathrm{~mm}$. O período mais seco (junho-setembro), possui temperaturas mais elevadas. O mês mais seco é agosto, chove cerca de somente $13 \mathrm{~mm}$. Uma peculiar característica das chuvas da cidade é por serem rápidas (normalmente não ultrapassam os 30 minutos) e bastante fortes (grande força da água e ventos). A incidência de raios também é muito comum (WIKIPÉDIA, 2011).

Os registros de casos de LTA do município são feitos com base nos resultados de exame de Intradermorreação de Montenegro, reação de imunofluorescência indireta (RIFI) e confirmada por exame parasitológico direto. O levantamento dos dados foi realizado a partir dos registros existentes na Secretaria Municipal de Saúde e do Centro de Controle de Zoonoses do município referente ao período de janeiro 2007 a outubro 2011.

Foi realizado também o levantamento de dados sobre a captura de flebotomíneos no período de setembro de 2006 a agosto de 2007, de quatro bairros segundo seu posicionamento cardial, sendo eles: Loteamento Boa Vista, Parque alvorada, Centro Operário e Parque Piauí. Os espécimes eram capturados, por agentes de saúde, com armadilhas luminosas do tipo CDC (Centers for Disease Control) instaladas no interior de domicílios e em abrigos de animais domésticos, na zona rural e periurbana, entre 18 e 6:00h de acordo com as recomendações do Manual de Vigilância da Leishmaniose Tegumentar Americana (2007). Os dados coletados foram tabulados em programa open Office, feitos médias e desvio padrão. 
ALVES, C.C.V., MAGALHÃES, N.A. e SOUSA, G.G.T. Incidência de Leishmaniose Tegumentar Americana no Município de Timon/MA. PUBVET, Londrina, V. 6, N. 30, Ed. 217, Art. 1449, 2012.

\section{Resultados e discussão}

Desde a descoberta do gênero Leishmania por Ross, em 1903, até o presente, houve uma mudança no aspecto epidemiológico da leishmaniose. Antes, a leishmaniose cutânea era considerada, basicamente, uma zoonose de animais selvagens, sendo transmitida acidentalmente ao homem quando este adentrava nas florestas para exploração, extração de madeira, abertura de estradas e mineração (CASTRO, 2002). Atualmente a doença apresenta um comportamento bastante diversificado acometendo pessoas em diferentes áreas geográficas, deixando de ser restrita a pena a áreas rural.

O município passou a realizar registros da LTA somente a partir do ano de 2007, entretanto já havia suspeita da doença ocorrer à bastante tempo. No período pesquisado foram registrados 60 casos. O ano de 2011 foi o que apresentou maior incidência com 22 casos registrados, somente até o mês de setembro, representando $36,67 \%$ de todas as ocorrências do município, no período pesquisado. As menores incidências ocorreram nos anos de 2008 e 2009 com seis casos (10\%) em cada ano. Em relação à distribuição por meses do ano o que se observa é que não há uma uniformidade que deve está relacionado com a grande variação do período de incubação do agente causador da LTA segundo descreve MARZOCHI (1992).

Em relação ao sexo, 66,7\% eram masculinos. Resultado semelhante ao de NUNES; PAULA (2006); CASTRO et al. (2002) e Ribeiro (2006), os quais justificam pelo fato de se tratar de uma doença ligada ao trabalho e de habitantes de áreas novas, recém abertas, para ampliação da zona urbana. Os casos registrados, no município, não ocorreram em áreas novas fato que pode ser explicado por ter havido a contaminação em outras áreas relacionada ao local de trabalho ser localizado em pontos favoráveis à epidemiologia da doença (Tabela 1). 
ALVES, C.C.V., MAGALHÃES, N.A. e SOUSA, G.G.T. Incidência de Leishmaniose Tegumentar Americana no Município de Timon/MA. PUBVET, Londrina, V. 6, N. 30, Ed. 217, Art. 1449, 2012.

Tabela 1. Incidência de LTA de acordo com sexo no período 2007 a 2011, no município de Timon - MA.

\begin{tabular}{lccccc}
\hline Anos & Masculino & Feminino & Total & Desvio Padrão & Média \\
\hline 2007 & 11 & 4 & 15 & 4,94 & 7,5 \\
2008 & 4 & 2 & 6 & 1,41 & 3 \\
2009 & 5 & 1 & 6 & 2,82 & 3 \\
2010 & 8 & 3 & 11 & 3,53 & 5,5 \\
2011 & 12 & 10 & 22 & 1,41 & 11 \\
\hline Total Geral & 40 & 20 & 60 & - & - \\
\hline
\end{tabular}

A média anual de casos entre os homens foi o dobro em relação à das mulheres, oito e quatro respectivamente, com desvio padrão de 3,53. LAINSON (1987) relata que a maior incidência é entre pessoas adultas, em atividade, do sexo masculino. No município os casos ocorreram mais em indivíduos com idade variando de 50 a 64 anos. Foram registrados 15 casos representando $25 \%$ da população em estudo (Tabela 2). Em função do período de incubação ser bastante variável, a ocorrência nessa faixa etária pode está relacionada com esse fato (BRASIL, 2000).

Tabela 2. Incidência de LTA, por faixa etária, no período de janeiro de 2007 a setembro de 2011, no município de Timon - MA.

\begin{tabular}{lcccccc}
\hline \multicolumn{7}{c}{ ANOS } \\
\hline Faixa Etária & $\mathbf{2 0 0 7}$ & $\mathbf{2 0 0 8}$ & $\mathbf{2 0 0 9}$ & $\mathbf{2 0 1 0}$ & $\mathbf{2 0 1 1}$ & Total \\
\hline >1 ano & 1 & - & - & - & - & 1 \\
5 a 9 & 1 & - & - & 2 & 3 & 6 \\
10 a 14 & - & - & - & 1 & 0 & 1 \\
20 a 34 & 6 & 1 & 2 & 1 & 2 & 12 \\
35 a 49 & - & 2 & - & 3 & 6 & 11 \\
50 a 64 & 3 & 2 & 1 & 2 & 7 & 15 \\
65 a 79 & 3 & 1 & 2 & 1 & 3 & 10 \\
$>80$ & 1 & - & 1 & 1 & 1 & 4 \\
\hline Total Geral & 15 & 6 & 6 & 11 & 22 & 60 \\
\hline
\end{tabular}

A média anual foi de 12 casos com desvio padrão de 6,75. Em relação à faixa etária a média anual foi de 7,5 casos com desvio padrão de 5,26 . Pode-se verificar que as maiores incidências foram no inicio e no final do período 
ALVES, C.C.V., MAGALHÃES, N.A. e SOUSA, G.G.T. Incidência de Leishmaniose Tegumentar Americana no Município de Timon/MA. PUBVET, Londrina, V. 6, N. 30, Ed. 217, Art. 1449, 2012.

pesquisado, equivalendo a cinco anos. A LTA apresenta um comportamento sazonal, apresentando picos a cada cinco anos (BRASIL, 2007).

A captura de flebotomíneos é um dos métodos utilizado para que se possa fazer um levantamento epidemiológico da doença e, assim, desenvolver estratégias para seu combate e controle. $O$ registro que se tem sobre captura desse inseto vetor refere-se ao período de agosto de 2006 a setembro de 2008, realizados nos bairros Loteamento Boa Vista, Parque Piauí, Centro Operário e Parque Alvorada (Tabela 3), onde foram identificadas as espécies: Lutzomyia longipalpis, L. lenti, L. evandroi, L. termitophila, L. goiana e L. migonei. Nesses há registros de casos de LTA tanto no período de captura como nos subsequentes até setembro de 2011. Das espécies capturadas todas são incriminadas como vetores, exceto a L. longipalpis. (BRASIL, 2007).

Tabela 3. Porcentagens de espécies de flebotomíneos capturados nos bairros monitorados do município de Timon-MA, no período de agosto de 2006 a setembro de 2008.

\begin{tabular}{lccc}
\hline Bairros & Anos & Longipalpis \% & Outros \% \\
\hline Loteamento Boa & 2007 & 99,8 & 0,2 \\
Vista & 2008 & 99,9 & 0,1 \\
Parque Piauí & 2007 & 99,9 & 0.1 \\
& 2008 & 99,9 & 0,1 \\
Centro Operário & 2007 & 99,6 & 0,4 \\
& 2008 & 99,7 & 0,3 \\
Parque Alvorada & 2007 & 99,7 & 0,3 \\
\hline Média & 2008 & 99,8 & 0,2 \\
\hline Desvio Padrão & - & 99,78 & 0,228 \\
\hline
\end{tabular}

A espécie encontrada em maior proporção foi a L.longipalpis com média de $99,8 \%$ e desvio padrão 0,11 da população flebotomínea existente no município de Timon. De acordo com Rebelo (1999) no estado do Maranhão as espécies mais encontradas com predominância no intra e no peridomicílios foram $L$. longipalpis $(67,4 \%$ e $70,2 \%$, respectivamente) e $L$. whitmani (31\% e $24,7 \%$, respectivamente) em estudo realizado nos municípios de Aldeias Altas, 
ALVES, C.C.V., MAGALHÃES, N.A. e SOUSA, G.G.T. Incidência de Leishmaniose Tegumentar Americana no Município de Timon/MA. PUBVET, Londrina, V. 6, N. 30, Ed. 217, Art. 1449, 2012.

Capinzal do Norte, Caxias, Codó, Coelho Neto, Timbiras, Timon e Tuntum. Nesse período de monitoramento não houve a presença de $L$. whitmani em Timon.

\section{Conclusão}

A ocorrência dos casos de LTA apresentou-se variável no período pesquisado, evidenciando-se picos no período de cinco anos.

A LTA ocorreu também em áreas onde não houve a captura de espécies de flebotomíneos que estão incriminados como vetores sugerindo a participação de outras espécies no ciclo.

\section{Referências bibliográficas}

ARAGÃO, H. B. Transmissão da Leishmaniose no Brasil pelo Phlebotomus intermedius. Brasil Médico, vol. 36, p. 129-130, 1922.

BASANO S. de A.; CAMARGO L. M. A., Leishmaniose tegumentar americana: histórico, epidemiologia e perspectivas de controle. Revista Brasileira de Entomologia. vol. 7 n. 3, 2004.

BRASIL - Manual De Controle Da Leishmaniose Tegumentar Americana. 5 a Edição (revisada). Acessória de Comunicação e Educação em Saúde NED/ASCOM/FUNASA Setor de Autarquias Sul, Quadra 4, BI. N, $5^{\circ}$ andar - Sala 515. 70058-902 - Brasília/DF - 2000

BRASIL. Manual de Vigilância da Leishmaniose Tegumentar Americana / Ministério da Saúde, Secretaria de Vigilância em Saúde. - $2^{a}$ ed. atual. - Brasília: Editora do Ministério da Saúde. 180 p.: il. - (Serie A. Normas e Manuais Técnicos) Brasil. Ministério da Saúde.

Secretaria de Vigilância em Saúde, 2007.

BRASIL. Doenças infecciosas e parasitárias. Ministério da Saúde. Secretária de Vigilância em Saúde, Departamento de Vigilância Epidemiológica. 8a ed. rev. - Brasília-DF. 448p. : II. (Série B. Textos Básicos de Saúde), 2010.

CARVALHO M. do S. L. de. et al., Flebotomíneos (Diptera: Psychodidae) em áreas de ocorrência de leishmaniose tegumentar americana no Distrito Federal, Brasil, 2006 a 2008. Epidemiol. Serv. Saúde, Brasília, v. 19, n. 3 p. 227-237, julho/setembro, 2010.

CASTRO et al., Estudo das Características Epidemiológicas e Clinicas de 332 Casos de Leishmaniose Tegumentar notificados na Região Norte do Estado do Paraná de 1993 a 1998.

Revista da Sociedade Brasileira de Medicina Tropical, artigo 35: p. 445-452, setembro/outubro, 2002. 
DEANE, L. M. et al. Observações sobre o combate ao phlebotomuslongipalpis pela dedetização dominiciliares em focos endêmicos de calazar no Ceará. Revista Brasileira de Malariologia e doenças tropicais, vol. 7, p. 135-141, 1955.

FIOCRUZ (FUNDAÇÃO OSVALDO CRUZ) et al. Capacitação de profissionais que atuam em sistemáticas de flebotomíneos, p. 8-9, 2004.

FNS (Fundação Nacional de Saúde). Relatório Anual. São Luís: Serviço de Epidemiologia/Coordenação Regional do Maranhão/Fundação Nacional de Saúde, 1997.

GONTIJO, B.; CARVALHO, M. L. R. de. Leishmaniose Tegumentar Americana. Revista da

Sociedade Brasileira de Medicina Tropical, vol. 36, n.1, p.71-80, janeiro/fevereiro, 2003.

IBGE, Censo demográfico do município de Timon-MA, 2010

http://www.ibge.gov.br/cidadesat/topwindow.htm?1 visualizado em 05/14/2011

LAINSON, R.; Shaw, J. J. Evolution, classification and geographical distribution. In : Peters W, Killick-Kendrick R (eds) The Leishmaniases in Biology and Medicine. Academic Press, London, vol.1, p. 1987.

LEISHMANIASIS. http://apps. who.int/tdr/svc/diseases/leishmaniasis Visualizado em: $04 / 12 / 2011$

MARZOCHI, M. C. A. Leishmanioses no Brasil: as leishmanioses tegumentares. Jornal Brasileiro de Medicina, [S. I.], vol. 63, p. 82-104, 1992.

MARZOCHI, M. C.; MARZOCHI, K. B. Proposta de uma classificação clínica simplificada para as leishmanioses tegumentares do novo mundo. Revista da Sociedade Brasileira de Medicina Tropical. vol. 27, p. 91, 1994.

MICHALICK, M. S. Gênero leishmania. In: Neves, D.P. Parasitologia Humana. São Paulo: Atheneu. 2002. p. 31-35.

MONTEIRO W. M. et. al., Distribuição geográfica e características epidemiológicas da leishmaniose tegumentar americana em áreas de colonização antiga do Estado do Paraná, Sul do Brasil. Cad. Saúde Pública, Rio de Janeiro, vol. 24 n. 6, p. 1291-1303, junho 2008.

MOREIRA, J. Distribuição geográfica do botão endêmico dos países quentes. Anais da Sociedade de Medicina da Bahia. Artigo. Gazeta Medica da Bahia, Salvador, vol.26, p.369374 , abril. 1895.

MURRAY, H. W. et al.; Advances in leishmaniasis. The Lancet. Vol. 366, p.1561-77, 2005.

NUNES, A. G.; PAULA, et al. Aspectos epidemiológicos da leishmaniose tegumentar americana em Varzelândia, Minas Gerais, Brasil. Cad. Saúde Pub., Rio de Janeiro, v. 22, n. 6, p. 1343-1347, Jun, 2006.

PESSÔA, S. B.; BARRETTO, M. P. Leishmaniose tegumentar americana. São Paulo: Ministério da Educação e Saúde - Serviço de documentação. 527p, 1948.

PISCOPO T. V.; AZZOPARDI, C. M. Leishmaniasis. Postgraduate Medical Journal. vol. 82, p. 649-657, 2006. 
RANGEL, E. F. et al. Infecção natural de Lutzomyia intermedia (Lutz \& Neiva, 1912) em área endêmica de leishmaniose tegumentar do Estado do Rio de Janeiro. Memórias do Instituto Oswaldo Cruz, vol. 79, p. 395-396, 1984.

RANGEL, E. F.; LAINSON R. Flebotomíneos do Brasil. Editora Fiocruz. 20ed. - 615.899. Av. Brasil, 4036 - $1^{\circ}$ andar - sala 112 - Manguinhos, Rio de Janeiro. 368p. 2003.

REBÊLO et al., Flebotomíneos (Diptera, Psychodidae) de área endêmica de leishmaniose na região dos cerrados, Estado do Maranhão, Brasil. Cad. Saúde Pública. vol.15 n.3 Rio de Janeiro July/Sept. 1999.

REBÊLO et al., Distribuição de Lutzomyia whitmani em fitorregiões do estado do Maranhão, Brasil. Revista Saúde Pública; v. 43, n. 6, p. 1070-4, 2009.

REITHINGER, R. et al; Cutaneous leishmaniasis. The Lancet Infectious Diseases. vol. 7, p: 581-96, 2007.

RIBEIRO L. A. et al., Leishmania tegumentar americana (LTA) em Teófilo Otoni, Minas Gerais: uma visão sócio-econômica. XI Encontro Latino Americano de Iniciação Científica e VII Encontro Latino Americano de Pós-Graduação - Universidade do Vale do Paraíba. p: 1676-1679, 2006.

SHERLOCK, I. A.; SHERLOCK, V. A. Sobre a infecção experimental de phlebotomus longipalpis pela leishmania donovani. Revista Brasileira de Biologia, vol. 21, p. 409-418, 1961.

SHERLOCK, I. A.; SHERLOCK, V. A. Tentativa de transmissão da leishmania donovani pela picada de lutzomyia longipalpis, entre cães. Revista da Sociedade Brasileira de Medicina Tropical, vol. 6, p. 35-39, 1972.

SMITH, R. O. A; HALDER, K. C.; AHMED, I. Further investigations on the transmission of kalaazar. Part III. The transmission of kala-azar by thebite of the sandfly P. argentipes. Indian Journal of Medical Research, vol. 28, p. 585-591, 1940.

SMITH, R. O. A; HALDER, K. C.; AHMED, I. Further investigations on the transmission of kalaazar. Part VI. A second series of L. donovani by P. argentipes. Indian Journal of Medical Research, vol. 29, p. 799-802,1941.

SPLENDORE, A. Bouba, blastomicose, leishmaniose: nota sobre algumas afecções framboesicas observadas no Brasil. Imprensa Médica, [S.I.], jan. 1911.

VALE, E. C. S. do; FURTADO T. Leishmaniose tegumentar no Brasil: revisão histórica da origem, expansão e etiologia. Anais Brasileiros de Dermatologia. vol. 80, n. 4, p. 421-8, 2005.

WALLBANKS, K R. Aphid derivedsugarsn is the neotropicsl sandfly Lutzomiya peruensis. Tropical Medicina and Parasitology. vol. 42, p.60-62, 1991

WIKIPÉDIA, Clima e localização do município de Timon-MA http://pt.wikipedia.org/wiki/Timon visualizado em 04/12/2011

ZANZARINI, P. D.; SANTOS, D. R.; SANTOS, A. R et al. Leishmaniose tegumentar americana canina em municípios do norte do Estado do Paraná, Brasil. Cad. Saúde Pública, vol.21, p.109-118, 2005. 